\title{
Determinantes sociales en la diferencial de mortalidad entre mujeres y hombres en Cuba
}

\author{
Social determinants found in the differences of mortality \\ indexes between females and men in Cuba
}

\author{
Ariadna Corral Martín '; I leana Elena Castañeda Abascal "; Manuel Barzaga \\ Torres "'I; María Cecilia Santana Espinosa'v \\ 'Especialista de I Grado en Medicina General Integral y Bioestadística. Instructor. \\ Escuela Nacional de Salud Pública. La Habana, Cuba. \\ "Doctora en Ciencias de la Salud. Profesor Titular. Escuela Nacional de Salud \\ Pública. La Habana, Cuba. \\ II'Especialista de I Grado en Medicina General Integral. Escuela Nacional de Salud \\ Pública. La Habana, Cuba. \\ IV Máster en Dirección. Profesor Auxiliar. Escuela Nacional de Salud Pública. La \\ Habana, Cuba.
}

\section{RESUMEN}

I ntroducción Los determinantes que diferencian biológica, sexual, cultural y socialmente a hombres y mujeres tienen implicaciones respecto a cómo y cuáles problemas de salud les afectan y sobre las intervenciones sanitarias que demandan. Objetivos Identificar diferencias en los niveles de mortalidad entre mujeres y hombres en la población cubana en el año 2006.

Métodos Se realizó un estudio descriptivo transversal utilizando las bases de datos de mortalidad de la Dirección Nacional de Estadísticas. Para obtener la información se calcularon las tasas de mortalidad específica por edad, sexo y años de vida potencialmente perdidos así como razones de tasas para las primeras causas de muerte.

Resultados Se encontró que los hombres tuvieron una sobremortalidad en la mayoría de las principales causas de muerte, dejando de vivir más prematuramente que las mujeres por un grupo de enfermedades crónicas, accidentes y lesiones autoinfligidas; la presencia de tumores malignos repercutió negativamente en ambos sexos.

Conclusiones Existen diferencias entre hombres y mujeres a favor de estas últimas en relación con los años de vida potencialmente perdidos, aunque la brecha entre ambos sexos muestra tendencia negativa para la mujer.

Palabras clave: Determinantes, mortalidad específica, años de vida potencialmente perdidos. 


\section{ABSTRACT}

I ntroduction The determinants that differentiate men and women from the biological, sexual, cultural and social viewpoints have a bearing on what kind of health problems affect them and how as well as on the required health interactions. Objectives To identify those differences in mortality indexes between Cuban men and women in 2006.

Methods A cross-sectional descriptive study using mortality index databases from the National Division of Statistics. Age-specific, sex-specific and potential years of life lost-specific mortality rates along with rate rations for the first causes of death were estimated to gather proper information.

Results It was found that men had an overmortality rate in most of the main causes of death, and died earlier than women from a group of chronic diseases, accidents, self-inflicted lesions; additionally, malignant tumors negatively affected both sexes.

Conclusions There are differences between men and women that favor the latter with respect to potential years of life lost, although the gap between men and women shows negative tendency towards the women.

Key words: Determinants, specific mortality, potential years of life lost.

\section{NTRODUCCI ÓN}

La visión de la salud desde los determinantes sociales con enfoque de género resulta exitosa para el diseño e implementación de acciones de salud, no solo porque visualiza la equidad como aspecto ético, sino también porque el género condiciona el perfil epidemiológico de la salud de las personas, el logro de objetivos sanitarios, la calidad y eficiencia de los servicios de salud.

La salud de mujeres y hombres es diferente y desigual. Diferente porque hay factores biológicos (genéticos, hereditarios, fisiológicos) que se manifiestan de forma disímil para cada sexo en relación con la salud y los riesgos de enfermedad, los que muchas veces se mantienen invisibles para los patrones androcéntricos de las ciencias de la salud. Desigual porque hay otros factores, que en parte son explicados por el género, y que influyen de una manera injusta en la salud de las personas. ${ }^{1-3}$

Las diferencias biológicas, sexuales, culturales y sociales entre los hombres y las mujeres tienen implicaciones respecto a cómo y cuáles son los problemas de salud que les afectan; por tanto, también en cuanto a sus necesidades de intervención sanitaria y social. ${ }^{3,4}$

Desde la determinante género, las diferencias de funciones, en cuanto a responsabilidades, acceso a recursos, información y poder, se reflejan en diferencias y desigualdades entre hombres y mujeres en riesgos y vulnerabilidad a 
la enfermedad y muerte, situación de salud, acceso a medidas preventivas y curativas, carga de enfermedad y calidad de la atención. ${ }^{2}$

Las diferencias injustas o iniquidades se producen a su vez, a partir de dos fuentes: la desigualdad de derechos y la de oportunidades. La desigualdad de derechos está relacionada con la justicia, se puede solucionar cuando existe la voluntad de crear un sistema de leyes dirigido hacia la igualdad entre mujeres y hombres mientras que la de oportunidades depende de los diferentes posicionamientos, condicionamientos y capacidades de cada ser humano en función de su forma de pensar y de vivir; su eliminación no se logra solo con la igualdad de derechos, al contrario, muchas veces se agrava; se logra con el compromiso social de ayudar a cada quien, de acuerdo a sus características particulares. ${ }^{5}$

La identificación de las diferencias de la mortalidad entre mujeres y hombres marca el camino para la determinación de los factores que conducen a esas brechas, todo lo cual es necesario para su disminución.

De este contexto surge una interrogante crucial: ¿cuáles fueron las diferencias existentes en la mortalidad de mujeres y hombres en Cuba en el 2006?

La noción de que existen diferencias intrínsecas entre los dos sexos se basa en la consideración de que la salud de las mujeres es solo reproductiva, y muchos profesionales sanitarios creen que la salud de las mujeres se relaciona solo con el embarazo, la anticoncepción, la planificación familiar y, recientemente, con la menopausia. ${ }^{6}$ (Bonino Méndez L. Masculinidad, salud y sistema sanitario. El caso de la violencia masculina. 2002).

Los estudios de género en el ámbito de la salud intentan responder preguntas relativas a la relación entre género, circunstancias sociales de las mujeres y los hombres y su estado de salud, para explicar las diferencias de morbilidad y mortalidad entre ellos, la dirección y magnitud de estas diferencias, que dependen en gran medida de cada problema concreto y de cada etapa del ciclo vital. ${ }^{7}$ (La salud pública desde la perspectiva de género y clase. En: Borrell C, García-Calvente MM, Martí JV, editores. Informe SESPAS).

El objetivo de este trabajo es identificar diferencias en los niveles de mortalidad según enfoque de género en la población Cubana en el año 2006.

\section{MÉTODOS}

Se realizó un estudio descriptivo transversal en Cuba en el año 2006. El universo estuvo constituido por toda la población de Cuba en el mencionado año.

Para la obtención de la información se utilizaron las bases de datos de mortalidad de la Dirección Nacional de Estadísticas para el año 2006, conformada con la información del Sistema de Información de Estadística de Defunciones y Defunciones Perinatales, codificada según la lista detallada de la $10^{\text {ma }}$ Revisión de la Clasificación Internacional de Enfermedades. Los datos de población se obtuvieron de las estimaciones elaboradas y suministradas por la Oficina Nacional de Estadísticas y disponibles en la Dirección Nacional de Estadística del Ministerio de Salud Pública.

Con el fin de medir las diferencias de la mortalidad entre los sexos, se calcularon las tasas específicas de mortalidad por grupo quinquenal de edad y sexo mediante 
el cociente conformado por el número de defunciones para el grupo de edad y sexo, tomando como denominador la población correspondiente a cada grupo de edad y sexo en el año 2006. Además, se calcularon las razones de tasas por sexo de las 10 primeras causas de muerte en el país para identificar sobremortalidad entre ambos sexos.

Para la mortalidad prematura se utilizó el indicador "años de vida potencialmente perdidos" (AVPP) para las primeras 10 causas de muerte y posteriormente se calculó el indicador para las 5 primeras causas con tasas más elevadas de AVPP, desagregadas por la variable sexo.

El cálculo de AVPP se realizó atendiendo al procedimiento conocido utilizando la siguiente expresión ( fórmula 1):

$$
A V P P=\Sigma \underbrace{\infty}_{x=0} d x\left(L_{x}-x\right)
$$

donde:

$\mathrm{L}_{\mathrm{x}}$ es la esperanza de vida a la edad $\mathrm{x}$ (el límite para la vida), $\mathrm{x}$ es la edad a la muerte $y \mathrm{dx}$ es el número de fallecidos a la edad $\mathrm{x}$.

Para medir la contribución de las cinco primeras causa de muerte al cambio observado en la esperanza de vida en el periodo analizado según sexo y grupos de edad, se calcularon los años de esperanza de vida perdidos (AEVP) utilizando el método planteado por Arriaga. ${ }^{8}$ Para aplicar este método se utilizaron tres de las funciones de la tabla de mortalidad abreviada calculada para el periodo estudiado, también se utilizó la esperanza de vida temporaria, que se obtiene a partir de estas funciones:

$\mathrm{I}_{\mathrm{x}}$ : número de supervivientes a la edad exacta $\mathrm{x}$.

${ }_{n} L_{x}$ : número de personas años vividos entre las edades $x$ y $x+n$.

$e_{\mathrm{x}}$ : esperanza de vida a la edad $\mathrm{x}$.

Los AEVP entre dos edades a y $b$, atribuibles a la mortalidad debida a la causa $z$ en el grupo de edad $[x, x+n]$, se obtienen mediante la expresión (fórmula 2):

$$
n^{A E V P_{x}}(z)={ }_{n} p_{x}(z)\left[\left(n+A_{x+n}\right)-n^{k}{ }_{x}\right]
$$

donde:

${ }_{n} p_{x}(z)$ es la proporción de fallecidos entre las edades $x$ y $x+n$ por la causa de muerte $z$ en la población estacionaria de la tabla de mortalidad.

$A_{x+n}$ es el promedio de años que los fallecidos entre $x$ y $x+n$ podrían haber vivido a partir de $x+n$ si no hubiesen muerto.

${ }_{n} \mathrm{k}_{\mathrm{x}}$ es el promedio de años vividos entre $x$ y $x+n$ por la población que fallece en ese grupo de edad, puesto que las defunciones se reparten por todo el grupo.

En resumen, la diferencia $\left(n+A_{x}+{ }_{n}\right)-{ }_{n} k_{x}$ da como resultado el promedio de años que la población fallecida entre $x$ y $x+n$ deja de vivir desde la edad $x$ (años que se podrían vivir - años realmente vividos). 


\section{RESULTADOS}

En el año 2006 el 15,4 \% de la población cubana tenía más de 60 años de edad, el $14,7 \%$ y el $16,1 \%$ de las personas mayores de esta edad correspondían al sexo masculino y femenino respectivante, con predominio de la población femenina debido a su mayor expectativa de vida.

En el propio año, se alcanzó una esperanza de vida al nacer de 78,9 años para mujeres y 75,1 para los hombres, con una diferencia de 3,8 años a favor de las mujeres. ${ }^{9}$ Resultados aunque favorables para la mujer, demuestran reducida brecha entre ambos sexos; otros autores informan diferencias entre 7 y 10 años.

La mortalidad en Cuba alcanzó una tasa general de 7,2 por 1000 habitantes en el 2006, con una sobremortalidad masculina que mostró una tasa de 7,8 fallecidos por cada 100 hombres, representando este sexo el 54,3\% del total de defunciones en el país, mientras que en las mujeres se registró una tasa de 6,6 fallecidas por 1000 féminas, aportando el $45,7 \%$ de todos los fallecidos.

En la tabla 1 se presentan las 10 primeras causas de muerte en Cuba en el 2006. Entre las principales causas de muerte ocurridas en ambos sexos se encuentran las enfermedades no trasmisibles que concentró el $71,5 \%$ de todas las defunciones; en el primer lugar, las enfermedades del corazón que contribuyeron con el 26,1 \% del total de las defunciones de las primeras 10 causas, seguida de los tumores malignos con el $15,4 \%$.

Los grupos de edad mayores de 50 años, tanto en hombres como en mujeres aportaron el mayor numero de fallecidos por estas causas, aunque en el caso de los tumores malignos se observó un incremento de las defunciones en edades más jóvenes, por ejemplo, el grupo de 30 a 49 años de edad alcanzó el 8,8 \% de las defunciones por tumores malignos, con predominio del sexo masculino (tabla 1 ). 
Tabla 1. Principales causas de muerte según sexo.

Cuba 2006

\begin{tabular}{|c|c|c|c|c|c|}
\hline \multirow{2}{*}{ Causas de muerte } & \multicolumn{2}{|c|}{ Hombres } & \multicolumn{2}{|c|}{ Mujeres } & \multirow{2}{*}{$\begin{array}{l}\text { Razón de } \\
\text { tasa } \\
\mathrm{H} / \mathrm{M}\end{array}$} \\
\hline & No. & Tasa & No. & Tasa & \\
\hline Enfermedades del corazón & 11364 & 201,2 & 9857 & 175,1 & 1,2 \\
\hline Tumores malignos & 11257 & 199,3 & 8438 & 149,9 & 1,3 \\
\hline Enfermedades cerebrovasculares & 4080 & 72,2 & 4267 & 75,8 & 1,0 \\
\hline Influenza y neumonía & 3191 & 56,5 & 2966 & 52,7 & 1,1 \\
\hline Accidentes & 2380 & 42,1 & 1696 & 30,1 & 1,4 \\
\hline $\begin{array}{l}\text { Enfermedades crónicas de las vías } \\
\text { respiratorias inferiores }\end{array}$ & 1519 & 26,9 & 1222 & 21,7 & 1,2 \\
\hline $\begin{array}{l}\text { Enfermedades de las arterias, arteriolas } \\
\text { y vasos capilares }\end{array}$ & 1474 & 26,1 & 1259 & 22,4 & 1,2 \\
\hline Diabetes mellitus & 748 & 13,2 & 1308 & 23,2 & 0,6 \\
\hline Lesiones autoinfligidas intencionalmente & 1100 & 19,5 & 269 & 4,8 & 4,1 \\
\hline $\begin{array}{l}\text { Cirrosis y otras enfermedades crónicas } \\
\text { del hígado }\end{array}$ & 720 & 12,7 & 299 & 5,3 & 2,4 \\
\hline
\end{tabular}

Fuente: Anuario Estadístico de Salud 2006. Dirección Nacional de Registro Médicos y Estadisticas de Salud.

Según sexo, los hombres presentaron una sobremortalidad en la mayoría de las primeras causas de muerte. Como se aprecia en la tabla 1, la razón de tasa de mortalidad por sexo muestra que los hombres tenían mayor riesgo de morir que las mujeres en todas las causas excepto por diabetes mellitus que las mujeres resultaron menos favorecidas. Se aprecia elevada mortalidad de los hombres por accidentes con respecto a las mujeres, por cada mujer que fallece por accidentes mueren aproximadamente 4 hombres; situación parecida tienen las muertes por cirrosis y otras enfermedades crónicas del hígado, donde el riesgo de morir en los hombres es 2 veces superior que en las mujeres.

Al observar la figura donde aparecen los años de vida perdidos prematuramente por todas las causas según edad y sexo, se puede analizar que los hombres dejan de vivir más años que las mujeres, las tasas de años perdidos aumentaron entre las edades de 50 a 55 años y alcanzaron su punto máximo alrededor de los 65 años para después descender en las edades más avanzadas, este comportamiento fue similar en ambos sexos.

En general, en Cuba se dejaron de vivir 637624 años por muertes prematuras, los hombres perdieron aproximadamente 26 años más que las mujeres, con una tasa de 72,8 años por cada 1000 hombres entre 0 y 74 años, mientras que las mujeres mostraron una tasa de 47,1 por 1000 mujeres en ese intervalo de edad.

En la tabla 2 se relacionan las principales causas de muerte por las que más se dejó de vivir en Cuba durante el año estudiado. Resultó interesante apreciar que los tumores malignos tuvieron un mayor impacto negativo en la salud de la población cubana según AVPP, ocuparon el primer lugar y desplazaron a las enfermedades del 
corazón con tasas de 17,6 y 17,0 por 1000 para el sexo masculino y femenino respectivamente, con ligeras diferencias entre ambos sexos aunque el sexo masculino resultó el más afectado. Las localizaciones en pulmón, próstata y colon, en el hombre y pulmón, mama y colon, en la mujer, aportaron la mayor mortalidad por tumores.

Los AVPP por muertes prematuras por enfermedades del corazón también repercutieron negativamente sobre los hombres con respecto a las mujeres con una tasa de 13,8 por 1000 y las mujeres con una tasa de 8,10 por 1000 .

Consecuentemente con los resultados observados en la mortalidad en relación con los accidentes, esta causa exhibió el diferencial mayor entre ambos sexos, pues por cada año que dejó de vivir una mujer, los hombres dejaron de vivir 4 años, sobre todo en edades más jóvenes, entre 30 y 49 años.

Las lesiones autoinfligidas presentaron un comportamiento parecido al que mostraron los accidentes con una razón de tasa de 3,8 desfavorable para los hombres (tabla 2).

Tabla 2. Años de vida potencialmente perdidos según primeras causas de muerte y sexo.

Cuba 2006

\begin{tabular}{|c|c|c|c|c|c|}
\hline \multirow[t]{3}{*}{ Causa de muerte } & \multicolumn{5}{|c|}{ Años de Vida Potencialmente Perdidos } \\
\hline & \multicolumn{2}{|c|}{ Hombres } & \multicolumn{2}{|c|}{ Mujeres } & \multirow{2}{*}{$\begin{array}{c}\text { Razón de tasa } \\
\mathrm{H} / \mathrm{M}\end{array}$} \\
\hline & No. & Tasa & No. & Tasa & \\
\hline Tumores & 94102 & 17,6 & 89887 & 17,0 & 1,0 \\
\hline Enfermedades del corazón & 73767 & 13,8 & 42685 & 8,10 & 1,7 \\
\hline Accidentes & 53700 & 10,1 & 13068 & 2,50 & 4,0 \\
\hline Enfermedades cerebrovasculares & 23920 & 4,50 & 18112 & 3,40 & 1,3 \\
\hline Lesiones auto infligidas & 24314 & 4,60 & 6097 & 1,20 & 3,8 \\
\hline
\end{tabular}

Fuente: Anuario Estadístico de Salud 2006. Dirección Nacional de Registros Médicos y

Estadisticas de Salud.

En la tabla 3 se muestra el aporte de las cinco causas de muerte que mayor impacto tuvieron en los AVPP al cambio en la esperanza de vida bajo el supuesto teórico de eliminar el riesgo de morir por estas.

El mayor incremento en las esperanzas de vida se produce cuando se elimina el riesgo de morir por tumores malignos, con lo cual puede aumentarse el valor de este indicador en más de 3 años. En esta enfermedad la mayor ganancia la aportó el sexo masculino con 4 años, mientras que el sexo femenino aportó 3,1 años de ganancia. La eliminación del riesgo de morir por enfermedades del corazón aumentó en 3,6 años el promedio de vida, igualmente los hombres aportaron más años que las mujeres. Por su parte los accidentes y enfermedades cerebrovasculares, si se erradicaran, contribuirían con una ganancia de 1 año de vida como promedio cada una y el sexo masculino aportaría más años que las mujeres (tabla 3). 
Tabla 3. Años de esperanza de vida ganados eliminando principales causas de muerte según sexo. Cuba 2006

\begin{tabular}{|c|c|c|c|}
\hline \multirow{2}{*}{ Causa de muerte } & \multicolumn{3}{|c|}{ Años de esperanza de vida ganados } \\
\hline & Hombres & Mujeres & Ambos sexos \\
\hline La cinco causas & 11,2 & 8,2 & 9,7 \\
\hline Tumores malignos & 4,0 & 3,1 & 3,8 \\
\hline Enfermedades del corazón & 3,6 & 2,7 & 3,1 \\
\hline Accidentes & 1,8 & 1,2 & 1,3 \\
\hline Enfermedades cerebrovasculares & 1,2 & 0,9 & 1,1 \\
\hline Lesiones autoinfligidas & 0,6 & 0,4 & 0,4 \\
\hline
\end{tabular}

Fuente: Anuario Estadístico de Salud 2006. Dirección Nacional de Registros

Médicos y Estadísticas de Salud.

\section{DISCUSIÓN}

Es conocida la paradójica mayor esperanza de vida de las mujeres, se reconoce que es aproximadamente de 7 a 10 años más dilatada que en los hombres, sin embargo, las mujeres presentan una incidencia más alta de morbilidad y de discapacidades durante su vida que los hombres, sobre todo porque acumulan muchas más enfermedades crónicas que los varones. Esto concuerda con la información encontrada en gran parte de los países, donde las mujeres tienen mayores probabilidades de experimentar el equivalente a dos o tres años más de mala salud, en comparación con los hombres durante el curso de su vida. ${ }^{10,11}$

Estimaciones de la OMS sugieren que las mujeres, tanto de países desarrollados como en desarrollo, pueden esperar vivir más años de vida con discapacidad y limitaciones funcionales que los hombres. ${ }^{12}$

En gran medida la mayor morbilidad y discapacidad de las mujeres se justifica por los patrones de socialización, roles familiares, obligaciones, expectativas laborales y tipos de ocupación que, frecuentemente, generan situaciones de sobrecarga física y emocional en las mujeres y que tienen una marcada influencia en su salud. Cabe mencionar que las mujeres durante el ciclo de vida realizan el trabajo de cuidadoras del bienestar familiar, puesto que son las que tienden a cuidar primero a los hijos, después a los padres y finalmente al cónyuge enfermo durante la vejez. ${ }^{13}$

En los últimos años los roles de las mujeres han cambiado, en muchos casos empeorando su situación al propiciar el fenómeno de la "doble jornada" o "triple jornada" (Madrid: Instituto de la Mujer, Ministerio de Trabajo y Asuntos Sociales. Disponible en: http://www.mtas.es/mujer/publicaciones/docs

/Atencion\%20biopsic\%20malestar.pdf). Esta diferencia de género, que se transforma en una desigualdad, condiciona seriamente el bienestar social, biológico, psicológico y físico de las mujeres de forma diferencial de acuerdo a su edad. 
Las enfermedades no transmisibles han acaparado las primeras causas de muerte sobre todo en la población de edad superior a 50 años en ambos sexos, en todo el mundo y Cuba no escapa a esta situación, con desventajas para los hombres con respecto a las mujeres en la mayoría de las causas de muertes, pero con una pobre brecha entre ambos. Corroborando estos resultados la OPS resalta que en el 2007 en América Latina y el Caribe, los AVPP en los hombres era $40 \%$ más alto que en las mujeres, este exceso de mortalidad de los hombres, sobre todo en los de mediana edad, se debe a mayores tasas de defunción por enfermedades crónicas. La sobremortalidad masculina alcanza proporciones dramáticas ( 5 a 50 veces mayor) que en la mujer, en relación con accidentes asociado al consumo excesivo de alcohol. ${ }^{14}$

Los factores sociales refuerzan la ventaja biológica de las mujeres, ya que los hombres resultan más expuestos a factores de riesgo, como el hábito de fumar o los peligros laborales; al mismo tiempo, existen comportamientos arriesgados asociados al "papel" masculino, que tiende a provocar conductas de aceptación del riesgo, un proceso que, en si mismo, guarda una estrecha relación con el género. Además, tradicionalmente, las mujeres usan los servicios de salud con mayor frecuencia que los hombres a lo largo de su vida, debido a las necesidades de atención derivadas de la reproducción y el cuidado de los hijos. Esta característica puede colocarlas en una posición ventajosa ante los hombres. ${ }^{15}$

Las conductas de género afectan la salud de los hombres, condicionando su vida personal y autoestima a las expectativas sociales de éxito laboral y económico, participan más que las mujeres en actividades que conllevan riesgos a la salud como ingerir alcohol, fumar cigarrillos, conducción agresiva de vehículos, comportamientos sexuales de riesgo y suicidio, a los que se les adiciona la falta de socialización para expresar sentimientos y padecimientos mentales y físicos. Estas situaciones pueden generar en los hombres condiciones cardiovasculares, accidentes laborales y de tránsito, cáncer, enfisema, problemas del hígado, depresión, ansiedad y angustia, ${ }^{3,4,14}$ que explicarían por qué los hombres presentaron tasas más elevadas de mortalidad por enfermedades cardiovasculares, accidentes y lesiones autoinfligidas. Los resultados de este estudio coinciden con otras investigaciones, según datos de la OMS, ${ }^{16}$ el $82,8 \%$ de los suicidios ocurridos en el año 2005 fueron de hombres, por 17,2 \% de mujeres, es decir, por cada mujer suicida hubo casi 5 hombres que incurrieron en este evento.

También puede achacarse al papel del género el exceso de carga de las muertes de los hombres por accidente y lesiones no intencionadas, como los accidentes de trabajo, que reflejan el papel histórico de los hombres como proveedores del hogar y su mayor tasa de empleo en las industrias más peligrosas.

Los tumores malignos generan la mayor cantidad de AVPP en Cuba desde la década del 90 desplazando a las enfermedades del corazón. En el último decenio, algunos países de América experimentaron una significativa disminución en los años de vida perdidos debido a muerte prematura por enfermedad coronaria, como por ejemplo Estados Unidos $(-76 \%)$, Argentina $(-51 \%)$, Canadá $(-50 \%)$ y Chile $(-23 \%),{ }^{17}$ mientras los tumores aumentaban constituyendo el problema de salud de mayor importancia mundialmente.

Es conocido que el cáncer es una enfermedad relacionada con el envejecimiento pero resulta alarmante que en el transcurso de los últimos años los tumores son una causa importante de mortalidad prematura en ambos sexos. 
Este rápido ascenso de los tumores como causa de muerte prematura en la población adulta está asociado, entre otras causas, a estilos de vida inadecuados. Un ejemplo lo constituye el cáncer de pulmón asociado al incremento del tabaquismo tanto en hombres como mujeres. Entre el 80 y $90 \%$ de los casos de cáncer de pulmón se atribuyen al tabaquismo, en el caso de Cuba, el $16 \%$ de mujeres eran fumadoras y el $14 \%$ de los hombres fumaban. En un estudio realizado en España en el año 2006 se estima que la proporción de mujeres fumadoras pasó del 10 en 1993 al $21 \%$ en el $2006 .{ }^{18}$ En este sentido, este estudio apunta que hasta mediados del siglo XX este hábito era exclusivo de los hombres, en parte debido al hecho de que el consumo de tabaco era y sigue siendo una práctica primordialmente de carácter social e iba ligado con el modelo de masculinidad tradicional, actualmente, el giro de este estereotipo, y la mayor inserción de la mujer en la sociedad, puede explicar cómo parte de las mujeres llegan a ser grandes fumadoras.

Por su parte, la eliminación total del riesgo de morir por cualquier de estas causas es como se mencionó anteriormente un supuesto teórico, que en la práctica difícilmente se lograría alcanzar. Lo que resulta importante es intervenir en los determinantes de salud y factores de riesgos relacionados con estas enfermedades y modificarlos para reducir en cierta magnitud el riesgo de morir por estas causas y desarrollar acciones de salud desde un enfoque de género.

Se puede concluir que en Cuba en el año 2006, las enfermedades crónicas no trasmisibles acapararon los primeros lugares entre las primeras causas de muerte ocurridas en ambos sexos, concentrando la mayor proporción de defunciones; los hombres exhibieron una sobremortalidad en la mayoría de las causas.

Los tumores malignos tuvieron el mayor impacto negativo en cuanto a los años que dejaron de vivir tempranamente los hombres y mujeres, asociado a estilos de vida inadecuados. Los accidentes y las lesiones autoinfligidas repercutieron negativamente en las muertes prematuras de los hombres en edades jóvenes, posiblemente ligadas a conductas de riesgos vinculadas a los roles sociales de género.

Se aprecia una brecha estrecha entre los AVPP en las mujeres y hombres en Cuba, lo que puede guardar relación, entre otras causas, con su participación en la mortalidad por tumores, al aumentar la tendencia en la mortalidad por cáncer de pulmón.

\section{REFERENCI AS BI BLI OGRÁFICAS}

1. Behar R, De La Barrera M, Michelotti J. Feminidad, masculinidad, androginidad y trastornos del hábito del comer. Rev Méd Chile 2002; 130:964-75.

2. Vega J, Bedregal $P$, Jadue L, Delgado I. Equidad de género en el acceso a la atención de salud en Chile. Rev Méd Chile. 2003; 131:669-78.

3. Rohlfs I, Borrell C, Fonseca M. Género, desigualdades y salud pública: conocimientos y desconocimientos. Gac Sanit. 2000; 14 (supl 3):60-71.

4. Krieger N. Gender, sexes and health: what are the connections-and why does it matter? Int J Epidemiol. 2003; 32:652-7. 
5. Castañeda Abascal IE. Reflexiones teóricas sobre las diferencias en salud atribuibles al género. Rev Cubana Salud Pública. 2007;33(2).

6. Bekker J. Investigating gender within health research is more than sex desegregations of data: a Multi-Facet and Health Model. Psychol Health Med. 2003; 8: 231-43.

7. Rohlfs I, De Andrés J, Artazcoz L, Ribalta M, Borrell C. Influencia del trabajo remunerado en el estado de salud percibido de las mujeres. Medicina Clínica (Barc. ). 1997; 108:566-71.

8. Arriaga EE. Los años de vida perdidos: su utilización para medir el nivel y cambio de la mortalidad. Notas de Población CELADE. 1996;24(63): 7-38.

9. Indicadores demográficos. Cuba y sus territorios. La Habana: Oficina Nacional de Estadísticas (ONE); 2007.

10. Rodríguez-Abrego G, Escobedo de la Peña J. Esperanza de vida saludable en la población mexicana. Perinatol Reprod Hum. 2006;20(1).

11. Boletín informativo. OCDE. 2003;2(7):8-9.

12. World Health Organization. Gender, health and aging [sitio en Internet]. 2003 [citado 14 Sept2006]. Disponible en: http://www.who.int/gender/documents/en/\%20Gender_Ageing.pdf

13. Salgado de Zinder N, Soc D, Wong R. Género y pobreza: determinantes de la salud en la vejez. Salud Pública Méx. 2007;49(supl 4).

14. Género, salud y desarrollo. Indicadores básicos. Washington, D.C.:OPS; 2007.

15. Wong R, Díaz JJ. Health care utilization among older Mexican: health and socioeconomic inequalities. Salud Pública Mex. 2007;49 supl 4.

16. Organización Mundial de la Salud. Informe sobre la salud en el mundo. Salud mental: nuevos conocimientos, nuevas esperanzas. Ginebra: OMS; 2005.

17. WHO. The World health report: 2004: Changing history. Geneva: WHO; 2004.

18. Begoña Iglesias A, Vázquez González F. Las mujeres y el tabaco: características ligadas al género. Revista Española Salud Pública. 2006; 74(1): 13.

Recibido: 28 de agosto de 2008.

Aprobado: 23 de octubre de 2008.

Ariadna Corral Martín. Escuela Nacional de Salud Pública. Calle Línea esq a I. EI Vedado 10 400. La Habana, Cuba.

E-mail: ariadna.corral@infomed.sld.cu 


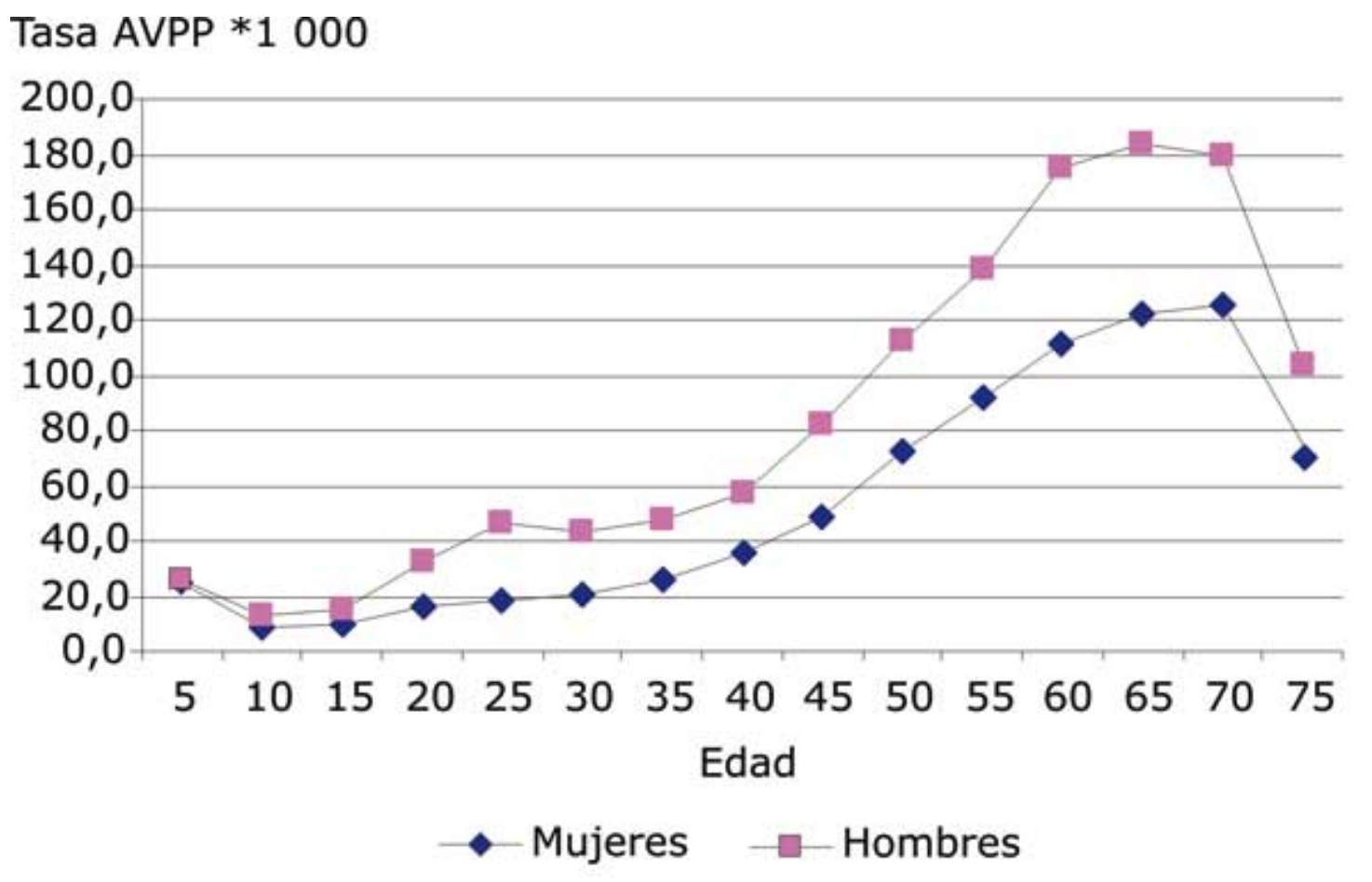

Fuente: Anuario Estadístico de Salud 2006. Dirección Nacional de Registros Médicos y Estadísticas de Salud.

Fig. Tasa de AVPP según grupos de edad y sexo. Cuba 2006. 